\title{
Matching for teams
}

\author{
G. Carlier $\stackrel{*}{\text { I. Ekeland }}{ }^{\dagger}$
}

October 7, 2007

\begin{abstract}
We are given a list of tasks $Z$ and a population divided into several groups $X_{j}$ of equal size. Performing one task $z$ requires constituting a team with exacly one member $x_{j}$ from every group. There is a cost (or reward) for participation: if type $x_{j}$ chooses task $z$, he receives $p_{j}(z)$; utilities are quasi-linear. One seeks an equilibrium price, that is, a price system that distributes all the agents into distinct teams. We prove existence of equilibria and fully characterize them as solutions to some convex optimization problems. The main mathematical tools are convex duality and mass transportation theory. Uniqueness and purity of equilibria are discussed. We will also give an alternative linear-programming formulation as in the recent work of Chiappori, McCann and Nesheim [2].
\end{abstract}

Acknowldegements: The authors are grateful to Robert J. McCann for suggesting to them the linear programming formulation of section 6 .

${ }^{*}$ Université Paris 9 Dauphine, CEREMADE, carlier@ceremade.dauphine.fr.

${ }^{\dagger}$ Canada Research Chair in Mathematical Economics, Department of Mathematics, University of British Columbia, ivar.ekeland@math.ubc.ca. 


\section{Introduction}

Consider a population divided into several equal groups $X_{j}, 0 \leq j \leq N$. We have to divide the total population $X=\cup X_{j}$ into teams, each team comprising exactly one member of each group. For $N=1$ this is the classical marriage problem: there is a group of men and a group of women, and they have to be paired one-to-one. As in the marriage problem, the matching will have to maximize some overall criterion for fitness.

This will be done by letting each individual pick a point $z$ in a set $Z$, independently of the others: more precisely, a set of prices (or costs) $p_{j}(z)$, $0 \leq j \leq N$, will be assigned to each $z$, and individuals in the category $X_{j}$ will pick their $z$ by maximizing a quasi-linear utility. Individuals which choose the same $z$ will be put in the same team.

The functions $p_{j}(z)$ will be found as the solutions of an optimization problem, so our result can be seen as giving a decentralized procedure for solving the matching problem. Alternatively, it can be seen as showing the existence of equilibrium prices in competitive markets where trading is not a two-sided interaction between buyer and seller, but requires the intervention of several types of agents. To build a new house, for instance, one has to hire (and pay) a wide variety of professionals (the architect) and tradesmen (the plumber, the mason), all of whom come in independently of the others and require market prices. Buying an existing house is not so simple a deal either: typically the buyer will have to borrow part of the money, so that the bank comes in as a third party into the deal. This approach was initiated in [3] for the case $N=1$ (the marriage problem), although the method used did not lend itself to such an extension.

The purpose of this paper is to generalize the results of [3] to the case $N \geq 2$ by means of convex duality arguments. Recently and independently, Chiappori, McCann and Nesheim in [2] formulated the matching problem as a linear programming problem and noticed that their approach also covers the multiple agents case. In section 6 , following a suggestion of Robert J. McCann, we will give a linear formulation of the problem as in [2].

The structure of the paper is as follows. Section 2 introduces the model; and in section 3 matching equilibria are defined. In section 4, we introduce two optimization problems which are naturally related to the matching problem. In section 5, we use convex duality to prove existence of equilibria and we give a variational characterization. An alternative formulation via linear programming is given in section 6 . Uniqueness and purity of equilibria are discussed in section 7. Finally, in section 8 , we extend our results to the case where a team does not necessarily consist of a finite number of agents but is given by a general measured space. 


\section{The model}

We consider a market where there is single, indivisible good which comes in different qualities $z \in Z$. In the sequel, we will refer to $Z$ as the quality space.

\subsection{Buyers/Consumers}

Each consumer buys one unit of the good. Consumer are heterogeneous: each of them is characterized by the value of some parameter $x_{0} \in X_{0}$, henceforth referred to as her type. Utilities are assumed to be quasi-linear with respect to prices: a consumer of type $x_{0}$ buying one unit of quality $z$ and paying $p_{0}$ derives the utility:

$$
u_{0}\left(x_{0}, z\right)-p_{0}
$$

Consumers' types are assumed to be distributed in the population according to some probability $\mu_{0}$ on some $\sigma$-algebra of $X_{0}$. We normalize the size of the population of consumers to 1 .

\section{$2.2 \quad$ Producers}

There are $N$ categories of producers denoted by $i=1, \ldots, N$. The production requires specialized labor from every category of producers. Specifically, to produce one unit of good (whatever its quality), one must assemble a team of one representative from each category, and each producer can participate in the production of one unit only.

Each category of producers is heterogeneous. In category $i$, each producer is characterized her type $x_{i} \in X_{i}$ (skill parameters say) and a cost function $c_{i}: X_{i} \times Z \rightarrow \mathbb{R}$. The population $X_{i}$ size is assumed to be 1 , and types are assumed to be distributed in the population according to some probability $\mu_{i}$ on some $\sigma$-algebra of $X_{i}$.

\subsection{Teams and nonlinear transfers}

For a good of a given quality to be traded, it is necessary in our model to gather a team consisting exactly of one consumer, corresponding to the additional category $j=0$, and one producer of each category $j=1, \ldots, N$. Note that this is consistent with our assumption that all the populations have the same size.

Given a tariff $z \in Z \rightarrow p_{0}(z) \in \mathbb{R}$, consumers of type $x_{0} \in X_{0}$ purchase qualities $z$ which solve the program:

$$
\sup _{z \in Z}\left\{u_{0}\left(x_{0}, z\right)-p_{0}(z)\right\} \text {. }
$$


Similarly, given a wage pattern $z \in Z \rightarrow w_{i}(z) \in \mathbb{R}$ for category $i=1, \ldots, N$, type $x_{i}$ producers offer qualities $z$ which minimize net cost:

$$
\inf _{z \in Z}\left\{c_{i}\left(x_{i}, z\right)-w_{i}(z)\right\}
$$

It will be convenient in the sequel not to distinguish between the consumers' category $(j=0)$ and the producers' categories $(j=1, \ldots, N)$ and to formulate everything in terms of costs and transfers. For notational conveniency, we therefore set

$$
c_{0}:=-u_{0}
$$

and for for $j=0, \ldots, N$ we define the transfer functions $\varphi_{j}$ by:

$$
\varphi_{0}(z)=-p_{0}(z), \varphi_{i}(z)=w_{i}(z), 1 \leq i \leq N
$$

For given transfers, optimal qualities for type $x_{j}$ are determined by the program

$$
\left.\varphi_{j}^{c_{j}}\left(x_{j}\right):=\inf _{z \in Z}\left\{c_{j}\left(x_{j}, z\right)-\varphi_{j}(z)\right)\right\} .
$$

which is the indirect utility which type $x_{j}$ derives from the transfer $\varphi_{j}$. In the sequel, we shall refer to $\varphi_{j}^{c_{j}}$ as the $c_{j}$-transform of the transfer function $\varphi_{j}$. Note that for every $\left(x_{j}, z\right) \in X_{j} \times Z$, one has the so-called Young's inequality

$$
\varphi_{j}^{c_{j}}\left(x_{j}\right)+\varphi_{j}(z) \leq c_{j}\left(x_{j}, z\right) .
$$

Let us also remark that, given the transfers $\varphi_{j}$ for category $j$, the demand set $D_{j}\left(x_{j}\right)$ for agents of type $x_{j}$, that is, the set of optimal qualities in problem (2), is defined by:

$$
D_{j}\left(x_{j}\right):=\left\{z \in Z \mid \varphi_{j}^{c_{j}}\left(x_{j}\right)+\varphi_{j}(z)=c_{j}\left(x_{j}, z\right)\right\} .
$$

Each team is assumed to be self-financed. In other words, in a team that produces $z$, the price paid by the consumer, $p_{0}(z)$, is the sum of the wages paid to the producers, $w_{i}(z)$ for $i=1, \ldots, N$. Equivalently, the transfers satisfy the balance condition

$$
\sum_{j=0}^{N} \varphi_{j}(z)=0, \forall z \in Z .
$$

Since we are dealing with a quality good, note that, even if the quality space has a linear structure, the transfers are inherently nonlinear. 


\subsection{Data and assumptions}

To sum up, the data of the model are the type spaces of each category $X_{0}, X_{1}, \ldots, X_{N}$, the probability distributions of types $\mu_{0}, \ldots, \mu_{N}$, and the cost functions $c_{0}, \ldots, c_{N}$ (recall that $c_{0}=-u_{0}$ ). Throughout the paper, we will assume the following:

- $X_{j}$ is a compact metric space equipped with its Borel $\sigma$-algebra for $j=0, \ldots, N$,

- $Z$ is a compact metric space

- $\mu_{j}$ is a Borel probability measure on $X_{j}$ for $j=0, \ldots, N$,

- $c_{j} \in C^{0}\left(X_{j} \times Z, \mathbb{R}\right)$ for $j=0, \ldots, N$.

We shall denote by $\Delta\left(X_{j}\right)$ the set of probabilities on $X_{j}$, so that $\mu_{j} \in$ $\Delta\left(X_{j}\right)$. For probabilities on product spaces, $\gamma_{j} \in \Delta\left(X_{j} \times Z\right)$, we denote by $\pi_{X_{j}} \gamma_{j} \in \Delta\left(X_{j}\right)$ and $\pi_{Z} \gamma_{j} \in \Delta(Z)$ respectively the first and second marginal of the joint probability $\gamma_{j}$. In other words, for every $f \in C^{0}\left(X_{j}, \mathbb{R}\right)$ and $g \in C^{0}(Z, \mathbb{R})$ one has:

$$
\int_{X_{j} \times Z}\left(f\left(x_{j}\right)+g(z)\right) d \gamma_{j}\left(x_{j}, z\right)=\int_{X_{j}} f\left(x_{j}\right) d \pi_{X_{j}} \gamma_{j}\left(x_{j}\right)+\int_{Z} f\left(x_{j}\right) d \pi_{Z} \gamma_{j}(z) .
$$

\section{$3 \quad$ Equilibria}

\subsection{Couplings and product lines}

Of course, there is not reason why the demand set $D_{j}\left(x_{j}\right)$ should be a singleton, that is, that the optimization problem (2) should have a unique solution. We will therefore allow agents of type $x_{j}$ to randomize their choices between the several optimal solutions. Alternatively, we can consider that not all agent of type $x_{j}$ pick the same optimal quality, so that there is for each element of $D_{j}\left(x_{j}\right)$ a certain proportion of agents which choose it

We will define a coupling $\gamma_{j}$ between the type space $X_{j}$ and the quality space $Z$ as any probability measure on the graph of $D_{j} \subset X_{j} \times Z$ which projects down to $\mu_{j}$ :

$$
\begin{aligned}
\pi_{X_{j}} \gamma_{j} & =\mu_{j} \\
\varphi_{j}^{c_{j}}\left(x_{j}\right)+\varphi_{j}(z) & =c_{j}\left(x_{j}, z\right) \quad \gamma_{j} \text {-a.s on } X_{j} \times Z
\end{aligned}
$$

For every Borel subsets $A_{j} \subset X_{j}$ and $B \subset Z$, we interpet $\gamma_{j}\left(A_{j} \times B\right)$ as the probability that an agent of category $j$ has her type in $A_{j}$ and an 
optimal quality in $B$. At equilibrium, the distribution of the demand for the quality good and the distribution of the supply for the quality good for each category should coincide, which means that

$$
\pi_{Z} \gamma_{j}=\nu
$$

for some $\nu \in \Delta(Z)$ which is independent of the category $j$. The probability $\nu \in \Delta(Z)$ will naturally be interpreted as a quality line. For $\nu \in \Delta(Z)$, we define

$$
\Pi\left(\mu_{j}, \nu\right):=\left\{\gamma \in \Delta\left(X_{j} \times Z\right) \mid \pi_{X_{j}} \gamma=\mu_{j}, \pi_{Z} \gamma=\nu\right\} .
$$

so that the equilibrium condition on the good market means that there is a product line $\nu$ such that

$$
\gamma_{j} \in \Pi\left(\mu_{j}, \nu\right), \quad j=0, \ldots, N .
$$

\subsection{Definition of matching equilibria}

An equilibrium consists of (quality dependent) transfers, (quality and type dependent) couplings and a probabilty measure on the quality space (the product line) such that:

- for each quality, the balance condition (5) is satisfied,

- consumers of each type choose maximizing utility qualities,

- producers of each category and each type choose minimizing cost qualities,

- there is equilibrium on the market for the good: the demand probability distribution equals the supply probability distribution for each category of producer's.

This gives the following precise definition

Definition $1 A$ matching equilibrium consists of a family of transfers $\varphi_{j} \in$ $C^{0}(Z, \mathbb{R})$, a family of probabilities $\gamma_{j} \in \Delta\left(X_{j} \times Z\right), j=0, \ldots, N$ and $a$ quality line $\nu \in \Delta(Z)$ such that:

1. For all $z \in Z$ :

$$
\sum_{j=0}^{d} \varphi_{j}(z)=0
$$

2. $\gamma_{j} \in \Pi\left(\mu_{j}, \nu\right)$ for every $j=0, \ldots, N$,

3. for every $j=0, \ldots, N$, one has:

$$
\varphi_{j}^{c_{j}}\left(x_{j}\right)+\varphi_{j}(z)=c_{j}\left(x_{j}, z\right) \quad \gamma_{j} \text {-a.s on } X_{j} \times Z
$$




\section{Two related optimization problems}

The aim of this section is to prove that matching equilibria are solutions of a certain optimization problem. To achieve this goal, we first need some basic results from optimal transportation theory that are recalled in the next paragraph. Indeed, the Monge-Kantorovich duality is of particular interest in our equilibrium context since the last two conditions in the definition of an equilibrium exactly are the extremality conditions for this duality.

\subsection{Mass transportation and Kantorovich Duality}

Given two compact metric spaces $X$ and $Z$, probability measures $\mu \in \Delta(X)$, $\nu \in \Delta(Z)$, and a cost function $c \in C^{0}(X \times Z, \mathbb{R})$, the Monge-Kantorovich optimal tranportation problem consists in finding a transport plan $\gamma$ with least cost:

$$
\left(\mathcal{M}_{\mu, \nu}\right) W_{c}(\mu, \nu):=\inf \left\{\int_{X \times Z} c(x, z) d \gamma(x, z): \gamma \in \Pi(\mu, \nu)\right\}
$$

Here $\Pi(\mu, \nu)$ denotes the set of probability measures on $X \times Z$ having $\mu$ and $\nu$ as marginals. If $\gamma \in \Pi(\mu, \nu)$ solves $\left(\mathcal{M}_{\mu, \nu}\right)$, it is called an optimal transportation plan between $\mu$ and $\nu$ for the cost $c$. Slightly abusing notations, we shall say that $\gamma$ solves $W_{c}(\mu, \nu)$.

It is customary in the Monge-Kantorovich theory to consider also the dual problem:

$$
\left(\mathcal{D}_{\mu, \nu}\right) \sup _{\varphi \in C^{0}(Z, \mathbb{R})}\left\{\int_{X} \varphi^{c}(x) d \mu(x)+\int_{Z} \varphi(z) d \nu(z)\right\} .
$$

where by definition $\varphi^{c}$ is the "c-concave transform" of $\varphi$. I it is defined for all $x \in X$ by:

$$
\varphi^{c}(x):=\inf _{z \in Z}\{c(x, z)-\varphi(z)\} .
$$

It is immediate to check that if $\varphi$ is bounded on $Z$, then $\varphi^{c} \in C^{0}(X, \mathbb{R})$. The main results from optimal transportation theory (we refer to [5] and [6] for proofs) that we shall need are summarized in the following:

Theorem 1 Assume $\mu \in \Delta(X), \nu \in \Delta(Z)$, and $c \in C^{0}(X \times Z, \mathbb{R})$. Then:

1. the supremum in $\left(\mathcal{D}_{\mu, \nu}\right)$ is attained by some $\varphi \in C^{0}(Z, \mathbb{R})$, the infimum in $\left(\mathcal{M}_{\mu, \nu}\right)$ is attained by some $\gamma \in \Pi(\mu, \nu)$,

2. the duality relation $W_{c}(\mu, \nu)=\inf \left(\mathcal{M}_{\mu, \nu}\right)=\sup \left(\mathcal{D}_{\mu, \nu}\right)$ holds, 
3. $\varphi$ solves $\left(\mathcal{D}_{\mu, \nu}\right)$ and $\gamma$ solves $\left(\mathcal{M}_{\mu, \nu}\right)$ if and only if:

$$
\varphi^{c}(x)=c(x, z)-\varphi(z) \quad \gamma \text {-a.e on } X \times Z
$$

In the Monge-Kantorovich problem, note that we allow to split the mass at $x \in X$ to different destinations $z \in Z$. Indeed, we can write a coupling $\gamma \in$ $\Pi(\mu, \nu)$ as $\gamma=\gamma^{x} \otimes \mu$ where $\gamma^{x}$ is the conditional probability of the destination $z$ given the source $x$. One may therefore view the Monge-Kantorovich as a stochastic problem where one source $x$ can be sent to several destinations $z$ according to a conditional probability $\gamma^{x}$. If one does not allow such a splitting of mass and imposes instead that $x$ is sent to a single destination $z=\sigma(x)$ (in other words if one imposes that $\gamma^{x}$ is the Dirac mass at $\sigma(x)$ or that $\gamma$ is supported by the graph of some function $\sigma: X \rightarrow Z$ ) then one obtains the so-called Monge problem. The requirement that the probability distribution $\nu$ is a prescribed target may be expressed by

$$
\sigma \sharp \mu=\nu
$$

where $\sigma \sharp \mu$ is the push-forward (or image measure) of $\mu$ through $\sigma$ and given by

$$
\sigma \sharp \mu(B):=\mu\left(\sigma^{-1}(B)\right)
$$

for every Borel subset $B$ of $Z$. A Borel $\sigma: X \rightarrow Z$ such that $\sigma \sharp \mu=\nu$ is called a transport map between $\mu$ and $\nu$. The Monge problem then reads as:

$$
\inf \left\{\int_{X} c(x, \sigma(x)) d \mu(x): \sigma \sharp \mu=\nu\right\} .
$$

Let us remark that transport maps between $\mu$ and $\nu$ may not exist; for instance, this is the case if $\mu$ is a Dirac mass whereas $\nu$ is not. Even when there exist transport maps, there may not exist an optimal one. In fact, the Monge problem is much more complicated than the (linear) MongeKantorovich problem and in general it does not admit solutions unless further assumptions are imposed on the data, especially on the cost function. More precisely, let us assume the following:

- $X=\bar{\Omega}$, with $\Omega$ an open bounded subset of $\mathbb{R}^{d}$,

- $\mu \in \Delta(X)$ is absolutely continuous with respect to the Lebesgue measure, such that $\mu(\partial \Omega)=0$,

- the cost function $c$ is continuous and Lipschitz "in $x$ uniformly in $z$ " (i.e. there exists a constant $C$ such that $\left|c\left(x_{1}, z\right)-c\left(x_{2}, z\right)\right| \leq C\left\|x_{1}-x_{2}\right\|$, $\left.\forall\left(x_{1}, x_{2}, z\right) \in X^{2} \times Z\right)$ 
- $c(., z)$ is differentiable on $\Omega$ for every $z \in Z$ and satisfies the Generalized Spence-Mirrlees condition:

$$
\text { if }\left(x, z_{1}, z_{2}\right) \in X \times Z^{2} \text { satisfy } \nabla_{x} c\left(x, z_{1}\right)=\nabla_{x} c\left(x, z_{2}\right) \text { then } z_{1}=z_{2} .
$$

Theorem 2 Under the conditions above, the Monge-Kantorovich problem $\left(\mathcal{M}_{\mu, \nu}\right)$ admits a unique solution $\gamma$ which is of the form $\gamma=(\mathrm{id}, \sigma) \sharp \mu$ where $\sigma$ is the unique (up to $\mu$-a.e. equivalence) solution of the Monge problem (7).

In other words, under the Spence-Mirrlees assumption (and the other regularity assumptions listed above), optimal transportation plans are unique and in fact given by an optimal transport map. We refer to [1] for a proof of this result. This will be useful when we will discuss uniqueness and purity of equilibria in section 7 .

Finally, let us note a duality result, which is much in the spirit of what is to come. Denote by $\Delta(Z)$ the space of Radon measures on $Z$, which is the dual of $C^{0}(Z, \mathbb{R})$. Define for all $\varphi \in C^{0}(Z, \mathbb{R})$ :

$$
F(\varphi):=-\int_{X} \varphi^{c}(x) d \mu(x) .
$$

Corollary $1 F$ is a convex continuous function on $C^{0}(Z, \mathbb{R})$. The Fenchel transform of $F$ is given for every $\nu \in \mathcal{M}(Z)$ by:

$$
F^{*}(\nu)= \begin{cases}W_{c}(\mu, \nu) & \text { if } \nu \in \Delta(Z) \\ +\infty & \text { otherwise. }\end{cases}
$$

As an immediate consequence, the function $\nu \longmapsto W_{c}(\mu, \nu)$ is convex and weakly $*$ l.s.c.. The proof is given in the Appendix.

\subsection{Optimization properties of equilibria}

Let us assume that $\left(\varphi_{j}, \gamma_{j}, \nu\right)$ is a matching equilibrium. It then follows from the last two conditions in the definition of an equilibrium and from Theorem 1 that each coupling $\gamma_{j}$ solves $\left(\mathcal{M}_{\mu_{j}, \nu}\right)$, and that $\varphi_{j}$ solves its dual $\left(\mathcal{D}_{\mu_{j}, \nu}\right)$. So, for all $j=0, \ldots, N$, one has:

$$
W_{c_{j}}\left(\mu_{j}, \nu\right)=\int_{X_{j} \times Z} c_{j}\left(x_{j}, z\right) d \gamma_{j}\left(x_{j}, z\right)=\int_{X_{j}} \varphi_{j}^{c_{j}} d \mu_{j}+\int_{Z} \varphi_{j} d \nu .
$$

Summing these equalities and using the balance condition (5) then yields:

$$
\sum_{j=0}^{d} W_{c_{j}}\left(\mu_{j}, \nu\right)=\sum_{j=0}^{d} \int_{X_{j}} \varphi_{j}^{c_{j}} d \mu_{j}
$$


Now let $\psi_{j} \in C^{0}(Z, \mathbb{R})$ be another balanced family of transfers:

$$
\sum_{j=0}^{N} \psi_{j}(z)=0, \forall z \in Z .
$$

The Monge-Kantorovich duality formula yields:

$$
W_{c_{j}}\left(\mu_{j}, \nu\right) \geq \int_{X_{j}} \psi_{j}^{c_{j}} d \mu_{j}+\int_{Z} \psi_{j} d \nu
$$

summing these inequalities and using (11) we then get:

$$
\sum_{j=0}^{d} W_{c_{j}}\left(\mu_{j}, \nu\right) \geq \sum_{j=0}^{d} \int_{X_{j}} \psi_{j}^{c_{j}} d \mu_{j}
$$

With (10), we deduce that the transfers $\varphi_{j}$ 's solve the following (concave) program:

$$
(\mathcal{P}) \sup \left\{\sum_{j=0}^{d} \int_{X_{j}} \varphi_{j}^{c_{j}} d \mu_{j}: \sum_{j=0}^{d} \varphi_{j}=0\right\} .
$$

Take some $\eta \in \Delta(Z)$. With the Monge-Kantorovich duality formula, the balance condition (5) and (10), we get

$$
\begin{aligned}
\sum_{j=0}^{d} W_{c_{j}}\left(\mu_{j}, \eta\right) & \geq \sum_{j=0}^{d}\left(\int_{X_{j}} \varphi_{j}^{c_{j}} d \mu_{j}+\int_{Z} \varphi_{j} d \eta\right) \\
& =\sum_{j=0}^{d} \int_{X_{j}} \varphi_{j}^{c_{j}} d \mu_{j}=\sum_{j=0}^{d} W_{c_{j}}\left(\mu_{j}, \nu\right)
\end{aligned}
$$

So that $\nu$ solves

$$
\left(\mathcal{P}^{*}\right) \inf \left\{\sum_{j=0}^{d} W_{c_{j}}\left(\mu_{j}, \nu\right): \nu \in \Delta(Z)\right\} .
$$

It turns out that this is a convex problem as a consequence of Corollary 1.

To sum up, at this point, we haven't proven anything about the existence of equilibria, but have discovered that if $\left(\varphi_{j}, \gamma_{j}, \nu\right)$ is a matching equilibrium then:

- the transfers $\varphi_{j}$ 's solve $(\mathcal{P})$,

- the quality line $\nu$ solves $\left(\mathcal{P}^{*}\right)$, 
- for each $j, \gamma_{j}$ solves $W_{c_{j}}\left(\mu_{j}, \nu\right)$.

Moreover in this case

$$
\min \left(\mathcal{P}^{*}\right)=\sum_{j=0}^{d} W_{c_{j}}\left(\mu_{j}, \nu\right)=\sum_{j=0}^{d} \int_{X_{j}} \varphi_{j}^{c_{j}} d \mu_{j}=\max (\mathcal{P}) .
$$

It follows from (14), that if a matching equilibrium exists then $(\mathcal{P})$ and $\left(\mathcal{P}^{*}\right)$ both possess solutions and achieve the same value. Hence a necessary condition for the existence of a matching equilibrium is:

$$
\max (\mathcal{P})=\min \left(\mathcal{P}^{*}\right) .
$$

We shall prove in the next section that this condition is fulfilled and actually sufficient for the existence of a solution. Indeed, programs $(\mathcal{P})$ and $\left(\mathcal{P}^{*}\right)$ are in fact dual problems in the usual sense of convex analysis (as developed for instance in Ekeland and Temam [4]).

\section{$5 \quad$ Existence and characterization of equilibria}

\subsection{Solving $(\mathcal{P})$ and $\left(\mathcal{P}^{*}\right)$}

In accordance with definition $(9)$ we set, for $\varphi \in C^{0}(Z, \mathbb{R})$ :

$$
F_{j}(\varphi):=-\int_{X_{j}} \varphi_{j}^{c_{j}} d \mu_{j}, \quad j=0, \ldots N .
$$

These are convex functions, and their inf-convolution $H$ (see [4] for instance) is defined by:

$$
H(\varphi)=\left(\square_{i=0}^{N} F_{j}\right)(\varphi):=\inf \left\{\sum_{j=0}^{N} F_{j}\left(\varphi_{j}\right): \sum_{j=0}^{N} \varphi_{j}=\varphi\right\}
$$

The inf-convolution is exact if the infimum is attained, that is, if for every $\varphi \in C^{0}(Z, \mathbb{R})$ there exists $\varphi_{0}, \ldots \varphi_{N}$ in $C^{0}(Z, \mathbb{R})$ such that

$$
\sum_{j=0}^{N} \varphi_{j}=\varphi \text { and } \sum_{j=0}^{N} F_{j}\left(\varphi_{j}\right)=H(\varphi) .
$$

It is well-known that $H$ is a convex function on $C^{0}(Z, \mathbb{R})$ and that the Legendre-Fenchel transform of $H$ is given by:

$$
H^{*}(\nu)=\left(\square_{i=0}^{N} F_{j}\right)^{*}(\nu)=\sum_{j=0}^{N} F_{j}^{*}(\nu)
$$


Following the standard arguments in [4], we rewrite $\left(\mathcal{P}^{*}\right)$ as:

$$
\inf _{\nu \in \mathcal{M}(Z)} \sum_{j=0}^{N} F_{j}^{*}(\nu)=-\left(\sum_{j=0}^{N} F_{j}^{*}\right)^{*}(0)=-H^{* *}(0) .
$$

Of course, by definition $\sup (\mathcal{P})=-H(0)$ hence $\sup (\mathcal{P})=\inf (\mathcal{P})$ provided $H(0)=H^{* *}(0)$, that is, $H$ is lower semi-continuous at 0 for the strong (or, since $H$ is convex, equivalently for the weak topology) of $C^{0}(Z, \mathbb{R})$. It is in fact the case as stated in the next proposition, whose proof can be found in the appendix.

Proposition 1 Let $H$ be defined by (15), then the following holds:

1. the infimal convolution is exact

2. $H$ is convex and lower semi-continuous, so that:

$$
H=H^{* *}=\left(\sum_{j=0}^{N} F_{j}^{*}\right)^{*} .
$$

The next result states that $(\mathcal{P})$ and $\left(\mathcal{P}^{*}\right)$ possess solutions and have the same value (no duality gap).

\section{Theorem 3}

$$
\max (\mathcal{P})=\min \left(\mathcal{P}^{*}\right)
$$

Proof. The fact that $\left(\mathcal{P}^{*}\right)$ possesses solutions is easy to see. Indeed, $\Delta(Z)$ is weakly $*$ compact in $\mathcal{M}(Z)$ and each function $\nu \rightarrow W_{c_{j}}\left(\mu_{j}, \nu\right)$ is l.s.c. for the weak $*$ topology. The fact that the supremum is attained in $(\mathcal{P})$ follows from proposition 1 (with $\varphi=0$ ). By proposition $1, H=H^{* *}$ so that in particular $\sup (\mathcal{P})=-H(0)=-H^{* *}(0)=\inf \left(\mathcal{P}^{*}\right)$.

\subsection{Existence and characterization of matching equi- libria}

Consider a family of transfers $\varphi_{j} \in C^{0}(Z, \mathbb{R})$, a family of probabilities $\gamma_{j} \in$ $\Delta\left(X_{j} \times Z\right), j=0, \ldots, N$ and a quality line $\nu \in \Delta(Z)$. We want to know if they consitute a matching equilibrium, in the line of definition 1 . With the existence and duality results of Theorem 3 at hand, we have the following characterization: 
Theorem $4\left(\varphi_{j}, \gamma_{j}, \nu\right)$ is a matching equilibrium if and only if:

- the functions $\varphi_{j}$ 's solve $(\mathcal{P})$,

- $\nu \operatorname{solves}\left(\mathcal{P}^{*}\right)$,

- for each $j=0, \ldots, N, \gamma_{j}$ solves $W_{c_{j}}\left(\mu_{j}, \nu\right)$.

Proof. The "only if" part has already been proven in paragraph 4.2. Assume now that the $\varphi_{j}$ 's $(\mathcal{P})$ solve, that $\nu$ solves $\left(\mathcal{P}^{*}\right)$ and that $\gamma_{j}$ solves $W_{c_{j}}\left(\mu_{j}, \nu\right)$ for every $j$. In particular this implies that the $\varphi_{j}$ 's are balanced and that $\gamma_{j} \in \Pi\left(\mu_{j}, \nu\right)$ for all $j$. Since $\gamma_{j}$ solves $W_{c_{j}}\left(\mu_{j}, \nu\right)$, one has

$$
W_{c_{j}}\left(\mu_{j}, \nu\right)=\int_{X_{j} \times Z} c_{j}\left(x_{j}, z\right) d \gamma_{j}\left(x_{j}, z\right) .
$$

using the fact that the $\varphi_{j}$ 's solve $(\mathcal{P})$, that $\nu$ solves $\left(\mathcal{P}^{*}\right)$, using $(17)$, the balance condition and $\gamma_{j} \in \Pi\left(\mu_{j}, \nu\right)$ we then get:

$$
\begin{aligned}
\sum_{j=0}^{N} \int_{X_{j} \times Z} c_{j}\left(x_{j}, z\right) d \gamma_{j}\left(x_{j}, z\right) & =\sum_{j=0}^{N} W_{c_{j}}\left(\mu_{j}, \nu\right) \\
& =\min \left(\mathcal{P}^{*}\right)=\max (\mathcal{P})=\sum_{j=0}^{d} \int_{X_{j}} \varphi_{j}^{c_{j}} d \mu_{j} \\
& =\sum_{j=0}^{d}\left(\int_{X_{j}} \varphi_{j}^{c_{j}} d \mu_{j}+\int_{Z} \varphi_{j} d \nu\right) \\
& =\sum_{j=0}^{d} \int_{X_{j} \times Z}\left(\varphi_{j}^{c_{j}}\left(x_{j}\right)+\varphi_{j}(z)\right) d \gamma_{j}\left(x_{j}, z\right) .
\end{aligned}
$$

One thus deduce from Young's inequalities (3) that for every $j$ one has

$$
\varphi_{j}^{c_{j}}\left(x_{j}\right)+\varphi_{j}(z)=c_{j}\left(x_{j}, z\right) \gamma_{j} \text {-a.s. on } X \times Z,
$$

which proves that $\left(\varphi_{j}, \gamma_{j}, \nu\right)$ is a matching equilibrium.

Since existence of solutions to $(\mathcal{P})$ and $\left(\mathcal{P}^{*}\right)$ has already been proven in Theorem 3 and existence of optimal plans for $W_{c_{j}}\left(\mu_{j}, \nu\right)$ follows from Theorem 1, we thus immediately deduce:

Proposition 2 There exist matching equilibria. 


\subsection{An example}

Let us illustrate the previous characterization results by a simple example. Consider the unidimensional case where $X_{j}=Z=[a, b]$ for all $j=0, \ldots, N$ and the cost function of category $j$ is

$$
c_{j}\left(x_{j}, z\right)=\lambda_{j}\left(\frac{1}{2} z^{2}-x_{j} z\right)
$$

where the $\lambda_{j}$ 's are positive constants that sum to 1 (this last condition is of course without loss of generality). Let us further assume for simplicity that $\mu_{j}$, the probability distribution of type $x_{j}$, is absolutely continuous with a positive density on $[a, b]$ for every $j$. Let $F_{j}$ be the cumulative distribution function of the type $x_{j}$. Our assumptions imply that $F_{j}$ is continuous and increasing on $[a, b]$ with $F_{j}(a)=0, F_{j}(b)=1$, hence $F_{j}$ is invertible with an increasing inverse $F_{j}^{-1}:[0,1] \rightarrow[a, b]$. In this case there is a unique monotone increasing function $\sigma_{j}$ such that $\sigma_{j} \sharp \mu_{0}=\mu_{j}$ (of course $\sigma_{0}=$ id) and $\sigma_{j}$ is given by the explicit formula $\sigma_{j}=F_{j}^{-1} \circ F_{0}$. Let us then define

$$
\bar{\sigma}:=\left(\sum_{j=0}^{N} \lambda_{j} \sigma_{j}\right) \sharp \mu_{0}, \bar{\gamma}_{j}:=\left(\sigma_{j}, \bar{\sigma}\right) \sharp \mu_{0} \text {, and } \bar{\nu}:=\bar{\sigma} \sharp \mu_{0} \text {, }
$$

and for all $j$ and $z \in[a, b]$,

$$
\bar{\varphi}_{j}(z):=\int_{a}^{z} \lambda_{j}\left(s-\sigma_{j} \circ \bar{\sigma}^{-1}(s)\right) d s .
$$

We claim that $\left(\bar{\varphi}_{j}, \bar{\gamma}_{j}, \bar{\nu}\right)$ is an equilibrium and that it is the only one (up to the addition of constants that sum to 0 to the $\bar{\varphi}_{j}$ 's) since the generalized Spence-Mirrlees condition is satisfied here for all the cost functions (see section 7 for a more detailed discussion). Indeed, by construction the family $\bar{\varphi}_{j}$ 's is balanced, $\bar{\gamma}_{j} \in \Pi\left(\mu_{j}, \bar{\nu}\right)$ solves $W_{c_{j}}\left(\mu_{j}, \bar{\nu}\right)$ (the support of $\bar{\gamma}_{j}$ is indeed included in the graph of a nondecreasing function and this ensures the optimality, see [6] for details) and it easy to check that:

$$
\bar{\varphi}^{c_{j}}\left(\sigma_{j}\left(x_{0}\right)\right)+\bar{\varphi}_{j}\left(\bar{\sigma}\left(x_{0}\right)\right)=\lambda_{j}\left(\frac{1}{2} \bar{\sigma}^{2}\left(x_{0}\right)-\sigma_{j}\left(x_{0}\right) \bar{\sigma}\left(x_{0}\right)\right), \forall x_{0} \in[a, b]
$$

so that $\bar{\varphi}^{c_{j}}\left(x_{j}\right)+\bar{\varphi}_{j}(z)=\lambda_{j}\left(\frac{1}{2} z^{2}-x_{j} z\right) \gamma_{j}$-a.s., which proves that $\left(\bar{\varphi}_{j}, \bar{\gamma}_{j}, \bar{\nu}\right)$ is a matching equilibrium. 


\section{Linear programming reformulation}

In [2], Chiappori, McCann and Nesheim gave a linear programming formulation of the hedonic price equilibrium problem. In this section, following a suggestion of Robert J. McCann, we prove that equilibrium product lines, that is, solutions $\nu$ of $\left(\mathcal{P}^{*}\right)$, can be obtained by solving a linear programming problem.

First define $X:=X_{0} \times \ldots \times X_{N}$ and for every, $x:=\left(x_{0}, \ldots, x_{N}\right) \in X$, the least cost:

$$
\bar{c}(x):=\bar{c}\left(x_{0}, \ldots, x_{N}\right):=\inf \left\{\sum_{j=0}^{N} c_{j}\left(x_{j}, z\right), z \in Z\right\} .
$$

For the sake of simplicity, let us assume that for every $x=\left(x_{0}, \ldots ., x_{N}\right) \in X$ there is a unique cost-minimizing quality $z=: \bar{z}(x)$ :

$$
\bar{c}(x)=\sum_{j=0}^{N} c_{j}\left(x_{j}, \bar{z}(x)\right) .
$$

Now let us consider the multi-marginal Monge-Kantorovich problem

$$
\inf _{\gamma \in \Pi\left(\mu_{0}, \ldots, \mu_{N}\right)} \int_{X} \bar{c}\left(x_{0}, \ldots, x_{N}\right) d \gamma\left(x_{0}, \ldots, x_{N}\right)
$$

where $\Pi\left(\mu_{0}, \ldots, \mu_{N}\right)$ denotes the set of probability measures on $X$ having $\mu_{0}, \ldots, \mu_{N}$ as marginals. Note that this is a linear programming problem in the variable $\gamma$.

The connection between the multi-marginal Monge-Kantorovich problem (18) and $\left(\mathcal{P}^{*}\right)$ is the following (the proof is given in the appendix):

Proposition 3 Under the previous assumptions, one has:

1. the infimum in (18) is attained and its value coincide with $\inf \left(\mathcal{P}^{*}\right)$,

2. if $\bar{\gamma}$ solves (18) then $\bar{\nu}:=\bar{z} \sharp \gamma$ solves $\left(\mathcal{P}^{*}\right)$,

3. if $\bar{\nu}$ solves $\left(\mathcal{P}^{*}\right)$ then there exists a solution of (18), $\bar{\gamma}$, such that $\bar{\nu}:=$ $\bar{z} \sharp \bar{\gamma}$. 


\section{On purity and uniqueness of equilibria}

This section is devoted to investigate when equilibria are unique, and when they are pure.. A pure equilibrium is an equilibrium in which agents of the same type and the same category all choose the same quality:

Definition 2 A matching equilibrium $\left(\varphi_{j}, \gamma_{j}, \nu\right)$ is called pure if and only if all the couplings $\gamma_{j}$ 's are of the form $\gamma_{j}=\left(\mathrm{id}, \sigma_{\mathrm{j}}\right) \sharp \mu_{\mathrm{j}}$ with $\sigma_{j}$ measurable $X_{j} \rightarrow Z$.

Note in particular that the previous definition implies that $\sigma_{j}$ is a transport map between $\mu_{j}$ and $\nu$ (i.e. $\sigma_{j} \sharp \mu_{j}=\nu$ ) but also (by Theorem 4) that it is an optimal transport between $\mu_{j}$ and $\nu$, meaning that it solves the Monge problem:

$$
\inf \left\{\int_{X_{j}} c_{j}(x, \sigma(x)) d \mu_{j}(x): \sigma \sharp \mu_{j}=\nu\right\} .
$$

It thus follows from Theorem 2 that if all the probabilities $\mu_{j}$ 's and all the cost functions $c_{j}$ 's satisfy the assumptions preceding Theorem 2 and in particular the generalized Spence-Mirrlees condition, then every matching equilibrium is in fact pure.

Now, for uniqueness:

Proposition 4 If some particular $j_{0}, \mu_{j_{0}}$ and $c_{j_{0}}$ satisfy the assumptions preceding Theorem 2, then the equilibrium product line $\nu$ is unique: problem $\left(\mathcal{P}^{*}\right)$ has a unique solution..

Proof. Indeed assume that $\nu$ and $\tau$ both solve $\left(\mathcal{P}^{*}\right)$ and let $\left(\varphi_{0}, \ldots, \varphi_{N}\right)$ be a solution of $(\mathcal{P})$. Let $\gamma_{j}$ and $\eta_{j}$ be respectively optimal transportation plans for $W_{c_{j}}\left(\mu_{j}, \nu\right)$ and $W_{c_{j}}\left(\mu_{j}, \tau\right)$. On the one hand, Theorem 4 tells us that $\left(\varphi_{j}, \gamma_{j}, \nu\right)$ and $\left(\varphi_{j}, \eta_{j}, \tau\right)$ are matching equilibria. On the other hand, Theorem 2 and our assumptions on category $j_{0}$, imply that $\gamma_{j_{0}}=\left(\mathrm{id}, \sigma_{\mathrm{j}_{0}}\right) \sharp \mu_{\mathrm{j}_{0}}$ and $\eta_{j_{0}}=\left(\mathrm{id}, \mathrm{t}_{\mathrm{j}_{0}}\right) \sharp \mu_{\mathrm{j} 0}$ for two maps $\sigma_{j_{0}}$ and $t_{j_{0}}$ which satisfy

$$
\nu=\sigma_{j_{0}} \sharp \mu_{j_{0}}, \tau=t_{j_{0}} \sharp \mu_{j_{0}} .
$$

By the definition of equilibria, for $\mu_{j_{0}}$-a.e. $x \in X_{j_{0}}$, the following holds

$$
\begin{aligned}
\varphi_{j_{0}}^{c_{j_{0}}}(x) & :=\inf _{z \in Z}\left\{c_{j_{0}}(x, z)-\varphi_{j_{0}}(z)\right\} \\
& =c_{j_{0}}\left(x, \sigma_{j_{0}}(x)\right)-\varphi_{j_{0}}\left(\sigma_{j_{0}}(x)\right) \\
& =c_{j_{0}}\left(x, t_{j_{0}}(x)\right)-\varphi_{j_{0}}\left(t_{j_{0}}(x)\right) .
\end{aligned}
$$


Our assumptions imply that $\varphi_{j_{0}}^{c_{j_{0}}}$ is Lipschitz continuous on $X_{j_{0}}$, and hence, by Rademacher's Theorem, differentiable $\mu_{j_{0}}$-a.e.

If $\varphi_{j_{0}}^{c_{j_{0}}}$ is differentiable at $x$ and the equalities above are satisfied at $x$ then one has:

$$
\nabla \varphi_{j_{0}}^{c_{j_{0}}}(x)=\nabla_{x} c_{j_{0}}\left(x, \sigma_{j_{0}}(x)\right)=\nabla_{x} c_{j_{0}}\left(x, t_{j_{0}}(x)\right)
$$

with the generalized Spence-Mirrlees condition this then yields

$$
\sigma_{j_{0}}=t_{j_{0}}, \mu_{j_{0}} \text {-a.s. }
$$

with (20) we then have $\nu=\tau$ which proves the uniqueness of the equilibrium product line.

\section{Extension to a general measured category space}

In this final section, we extend our main results to the case of a general measured category space that we denote $\Theta_{0}:=\Theta \cup\{0\}$ where $\theta=0$ again corresponds to the consumers' population and $\Theta$ is the space of producers' categories. We assume that $\Theta_{0}$ is a compact metric space equipped with a nonnegative measure $m \in \Delta\left(\Theta_{0}\right)$ of the form $m_{0}=m+\delta_{0}$. As before, the quality space is denoted $Z$ and assumed to be a compact metric space. We also suppose that there is a unique characteristic (compact metric) space $X$ for both consumers and producers of each category $\theta$. The category $\theta$ is characterized by a cost function $c_{\theta} \in C^{0}(X \times Z, \mathbb{R})$ (with the interpretation that $c_{0}=-u_{0}$, the opposite of the consumers'utility function) and a distribution of type given by a probability $\mu_{\theta} \in \Delta(X)$. It is assumed that for a given quality to be produced, a consumer and a team of producers of the different sectors has to be formed, drawn according to the measure $m$. Of course, the finite case where $\Theta_{0}=\{0, \ldots, N\}$ and $m_{0}=\sum_{j=0}^{N} \delta_{j}$ corresponds to the case studied in the previous sections.

We will also assume:

- $\left(\mu_{\theta}\right)_{\theta \in \Theta_{0}}$ is a Borel family of probability measures on $X$, (i.e. $\theta \rightarrow$ $\int_{X} f(x) d \mu_{\theta}(x)$ is Borel for every Borel bounded $\left.f: X \rightarrow \mathbb{R}\right)$,

- joint continuity of the cost, i.e. $(\theta, x, z) \in \Theta_{0} \times X \times Z \rightarrow c_{\theta}(x, z) \in$ $C^{0}\left(\Theta_{0} \times X \times Z, \mathbb{R}\right)$.

Before going further let us recall the definition of a Caratheodory function: 
Definition 3 Let $Y$ a compact metric space and let $(\theta, y) \mapsto f_{\theta}(y)$ be a realvalued function defined on $\Theta_{0} \times Y,\left(f_{\theta}\right)_{\theta \in \Theta_{0}}$ is a Carathéodory function on $\Theta_{0} \times Y$ if:

- for $m_{0}$-almost every $\theta \in \Theta_{0}, f_{\theta}$ is a continuous function on $Y$,

- for all $y \in Y, \theta \mapsto f_{\theta}(y)$ is measurable on $\Theta_{0}$.

We shall denote by $\mathcal{A}\left(\Theta_{0} \times Y\right)$ the set of bounded Carathéodory functions on $\Theta_{0} \times Y$.

It is well-known that if $\left(f_{\theta}\right)_{\theta \in \Theta_{0}} \in \mathcal{A}\left(\Theta_{0} \times Y\right)$ and $\left(p_{\theta}\right)_{\theta \in \Theta_{0}}$ is a Borel family of probability measures on $Y$ then the map $\theta \mapsto \int_{Y} f_{\theta}(y) d p_{\theta}(y)$ is measurable.

The (unknown) transfers will be given by a family $\left(\varphi_{\theta}\right)_{\theta \in \Theta_{0}} \in \mathcal{A}\left(\Theta_{0} \times Z\right)$. The interpretation is again that $\varphi_{0}(z)=-p_{0}(z)$ (with $p_{0}(z)$ the price of the quality good $z$ ) and $\varphi_{\theta}(z)$ is the wage paid to producers of category $\theta \in \Theta$ for producing $z$. The self-financing budget constraint of the team then reads as:

$$
\int_{\Theta_{0}} \varphi_{\theta}(z) d m_{0}(\theta)=0, \text { for all } z \in Z \text {. }
$$

The natural definition of an equilibrium in this context then reads as:

Definition 4 A matching equilibrium $\left(\left(\varphi_{\theta}\right)_{\theta \in \Theta_{0}},(\gamma)_{\theta \in \Theta_{0}}, \nu\right)$ consists of a bounded Carathédory function $\left(\varphi_{\theta}\right)_{\theta \in \Theta_{0}} \in \mathcal{A}\left(\Theta_{0} \times Z\right)$, a probability measure $\nu \in \Delta(Z)$ and a family $(\gamma)_{\theta \in \Theta_{0}}$ of elements of $\Delta(X \times Z)$ such that:

1. For all $z \in Z$ :

$$
\int_{\Theta_{0}} \varphi_{\theta}(z) d m_{0}(\theta)=0
$$

2. $\gamma_{\theta} \in \Pi\left(\mu_{\theta}, \nu\right)$ for $m_{0}$-almost every $\theta \in \Theta_{0}$,

3. for $m_{0}$-almost every $\theta \in \Theta_{0}$, one has:

$$
\varphi_{\theta}^{c_{\theta}}(x)=c_{\theta}(x, z)-\varphi_{\theta}(z) \gamma_{\theta} \text {-almost everywhere on } X \times Z \text {. }
$$

To prove existence and give a characterization of equilibria, one may follow the same strategy as in the discrete case, by considering the two optimization problems:

$$
(\mathcal{P}) \sup _{\left(\varphi_{\theta}\right)_{\theta \in \Theta_{0}} \in \mathcal{E}(0)} \int_{\Theta_{0}}\left(\int_{X} \varphi_{\theta}^{c_{\theta}}(x) d \mu_{\theta}(x)\right) d m_{0}(\theta)
$$


with

$$
\mathcal{E}(0):=\left\{\left(\varphi_{\theta}\right)_{\theta \in \Theta_{0}} \in \mathcal{A}\left(\Theta_{0} \times Z\right): \int_{\Theta_{0}} \varphi_{\theta}(z) d m_{0}(\theta)=0 \text { for all } z \in Z\right\} .
$$

and its dual:

$$
\left(\mathcal{P}^{*}\right) \inf _{\nu \in \mathcal{M}_{+}^{1}(Z)} \int_{\Theta_{0}} W_{c_{\theta}}\left(\mu_{\theta}, \nu\right) d m_{0}(\theta) .
$$

Of course, there are measure-theoretic subtleties (like the measurability of $\left.\theta \rightarrow W_{c_{\theta}}\left(\mu_{\theta}, \nu\right)\right)$ and specific difficulties to generalize Proposition 1 , but the main results basically are the same as in the finite case:

Theorem 5 1. Both $(\mathcal{P})$ and $\left(\mathcal{P}^{*}\right)$ possess solutions and

$$
\max (\mathcal{P})=\min \left(\mathcal{P}^{*}\right),
$$

2. let $\left(\varphi_{\theta}\right)_{\theta \in \Theta_{0}} \in \mathcal{A}\left(\Theta_{0} \times Z\right), \nu \in \Delta(Z)$ and $\left(\gamma_{\theta}\right)_{\theta \in \Theta_{0}}$ be a family of elements of $\Delta(X \times Z)$, then $\left(\left(\varphi_{\theta}\right)_{\theta \in \Theta_{0}},\left(\gamma_{\theta}\right)_{\theta \in \Theta_{0}}, \nu\right)$ is a matching equilibrium if and only if:

(a) $\left(\varphi_{\theta}\right)_{\theta \in \Theta_{0}}$ solves $(\mathcal{P})$,

(b) $\nu$ solves $\left(\mathcal{P}^{*}\right)$,

(c) for $m_{0}$-almost every $\theta \in \Theta_{0}, \gamma_{\theta}$ is an optimal transportation plan between $\mu_{\theta}$ and $\nu$ for the cost $c_{\theta}$ i.e.:

$$
W_{c_{\theta}}\left(\mu_{\theta}, \nu\right)=\int_{X \times Z} c_{\theta}(x, z) d \gamma_{\theta}(x, z) .
$$

In particular, there exists matching equilibria.

The proof which is omitted here may be obtained from the authors upon request.

\section{Appendix}

\section{On $c$-concave analysis}

Let $X$ and $Z$ be two compact metric spaces. For $c \in C^{0}(X \times Z, \mathbb{R})$ and $\varphi \in C^{0}(Z, \mathbb{R})$, the $c$-concave transform of $\varphi$ is the function $\varphi^{c}$ defined on $X$ by formula (6), the $c$-concave envelope of $\varphi$ is the function denoted $\varphi^{c c}$ defined by:

$$
\varphi^{c c}(z):=\inf _{x \in X}\left\{c(x, z)-\varphi^{c}(x)\right\} \text { for all } z \in Z \text {. }
$$


We then have the following properties (we refer to [1] or [3] for proofs):

$$
\varphi^{c c} \geq \varphi \text { on } Z,\left(\varphi^{c c}\right)^{c}=\varphi^{c} \text { on } X .
$$

Let us denote by $d$ the distance on $Z$, and by $\omega_{c}$ the modulus of continuity of $c$ with respect to its second argument:

$$
\omega(t):=\sup \left\{\left|c\left(x, z_{1}\right)-c\left(x, z_{2}\right)\right|\left(x, z_{1}, z_{2}\right) \in X \times Z^{2}, d\left(z_{1}, z_{2}\right) \leq t\right\}
$$

since $X \times Z$ is compact $\omega_{c}(t)$ tends to 0 as $t \rightarrow 0^{+}$. Regularity of $c$-concave envelopes is guaranteed by the following:

Lemma 1 For all $z_{1}, z_{2} \in Z^{2}$ we have:

$$
\left|\varphi^{c c}\left(z_{1}\right)-\varphi^{c c}\left(z_{2}\right)\right| \leq \omega_{c}\left(d\left(z_{1}, z_{2}\right)\right) .
$$

This proves that the family $\left\{\varphi^{c c}, \varphi \in C^{0}(Z, \mathbb{R})\right\}$ is uniformly equicontinuous on $Z$. Similarly, $\left\{\varphi^{c}, \varphi \in C^{0}(Z, \mathbb{R})\right\}$ is uniformly equicontinuous on $X$.

\subsection{Proof of Corollary 1}

The claim of continuity immediately follows from the contraction property:

$$
\left\|\varphi_{1}^{c}-\varphi_{2}^{c}\right\|_{\infty, X} \leq\left\|\varphi_{1}-\varphi_{2}\right\|_{\infty, Z}, \forall\left(\varphi_{1}, \varphi_{2}\right) \in\left(C^{0}(Z, \mathbb{R})\right)^{2} .
$$

The claim of convexity immediately follows from the definition (6) which implies that for fixed $x \in X, \varphi^{c}(x)$ is a concave function of $\varphi$.

Let $\nu \in \mathcal{M}(Z)$. By the definition of the Fenchel conjugate:

$$
F^{*}(\nu)=\sup _{\varphi \in C^{0}(Z, \mathbb{R})}\left\{\int_{X} \varphi^{c}(x) d \mu(x)+\int_{Z} \varphi(z) d \nu(z)\right\}
$$

If $\nu$ is not a non-negative measure, there exists $\varphi \leq 0$ such that $\int_{Z} \varphi d \nu>$ 0 . For every $t>0$, we then have $\left(t \varphi^{c}\right) \geq \min _{X \times Z} c$, hence:

$$
F^{*}(\nu) \geq \sup _{t>0}\left\{t \int_{Z} \varphi d \nu\right\}+\min _{X \times Z} c=+\infty .
$$

If $\nu(Z) \neq 1=\mu(X)$, we get:

$$
F^{*}(\nu) \geq \sup _{t \in \mathbb{R}}\{t(\nu(Z)-\mu(X))\}+\min _{X \times Z} c=+\infty .
$$

Finally, if $\nu \in \Delta(Z)$, using the duality relation of Theorem 1 we get $F^{*}(\nu)=$ $W_{c}(\mu, \nu)$. 


\section{Proof of proposition 1}

Let us prove first that the infimal convolution is exact. Let $\left(\varphi_{0}^{k}, \ldots, \varphi_{N}^{k}\right)_{k}$ be a minimizing sequence for the minimization problem (15) defining $H(\varphi)$. For $j=1, \ldots, N$ define

$$
\psi_{j}^{k}:=\left(\varphi_{j}^{k}\right)^{c_{j} c_{j}}
$$

and

$$
\psi_{0}^{k}:=\varphi-\sum_{j=1}^{N} \psi_{j}^{k} .
$$

By construction $\left(\psi_{0}^{k}, \ldots, \psi_{N}^{k}\right)$ is admissible for $H(\varphi), \psi_{j}^{k} \geq \varphi_{j}^{k}$ and $\left(\psi_{j}^{k}\right)^{c_{j}}=$ $\left(\varphi_{j}^{k}\right)^{c_{j}}$ for $j=1, \ldots, N$, hence we have

$$
\psi_{0}^{k}=\varphi-\sum_{j=1}^{N} \psi_{j}^{k} \leq \varphi-\sum_{j=1}^{N} \varphi_{j}^{k}=\varphi_{0}^{k}
$$

and since the $c_{0}$-transform is order reversing, we obtain $\left(\psi_{0}^{k}\right)^{c_{0}} \geq\left(\varphi_{0}^{k}\right)^{c_{0}}$. Thus, we get

$$
\sum_{j=0}^{N} F_{j}\left(\psi_{j}^{k}\right) \leq \sum_{j=0}^{N} F_{j}\left(\varphi_{j}^{k}\right)
$$

so that $\left(\psi_{0}^{k}, \ldots, \psi_{N}^{k}\right)_{k}$ is also a minimizing sequence for the minimization problem (15). Since the problem (15) is invariant by adding to the $\varphi_{j}$ constants that sum to 0 , there is no loss of generality in assuming that $\min _{Z} \psi_{j}^{k}=0$ for every $j=1, \ldots, N$. For $j=1, \ldots, N$ defining $\omega_{j}$ the modulus of continuity of $c$ with respect to its second argument:

$$
\omega_{j}(t):=\sup \left\{\left|c_{j}\left(x_{j}, z_{1}\right)-c\left(x_{j}, z_{2}\right)\right|\left(x_{j}, z_{1}, z_{2}\right) \in X_{j} \times Z^{2}, d\left(z_{1}, z_{2}\right) \leq t\right\}
$$

and using lemma 1 we then get:

$$
0 \leq \psi_{j}^{k} \leq M_{j}:=\omega_{j}(\operatorname{diam}(Z)) \text { on } Z \text {, for } j=1, \ldots, N \text {. }
$$

With Lemma 1, the previous bounds and (26), we deduce that the sequence $\left(\psi_{j}^{k}\right)_{k}$ is bounded and uniformly equicontinuous for every $j=0, \ldots, N$. By Ascoli's theorem, we may therefore assume, taking subsequences if necessary, that each $\psi_{j}^{k}$ converges as $k \rightarrow+\infty$ in $C^{0}(Z, \mathbb{R})$ to some $\varphi_{j}$. By the continuity of $F_{j}$ (see corollary 1) we immediately deduce that

$$
\sum_{j=0}^{N} \varphi_{j}=\varphi \text { and } \sum_{j=0}^{N} F_{j}\left(\varphi_{j}\right)=H(\varphi) .
$$


so the inf-convolution is exact, as announced. Moreover, let us remark that the $\varphi_{j}$ 's obtained above satisfy

$$
0 \leq \varphi_{j} \leq M_{j},\left|\varphi_{j}\left(z_{1}\right)-\varphi_{j}\left(z_{2}\right)\right| \leq \omega_{j}\left(d\left(z_{1}, z_{2}\right)\right), \forall\left(z_{1}, z_{2}\right) \in Z^{2}, j=1, \ldots, N .
$$

Now it remains to prove that $H$ is l.s.c (convexity is obvious). Assume that a sequence $\left(\varphi^{k}\right)_{k}$ converges to some $\varphi$ in $C^{0}(Z, \mathbb{R})$, then one can find continuous functions $\left(\varphi_{0}^{k}, \ldots, \varphi_{N}^{k}\right)$ that satisfy the estimates (28) and such that

$$
\sum_{j=0}^{N} \varphi_{j}^{k}=\varphi^{k} \text { and } \sum_{j=0}^{N} F_{j}\left(\varphi_{j}^{k}\right)=H\left(\varphi^{k}\right) .
$$

Thanks to (28) and Ascoli's Theorem again, we may assume, up to some subsequence, that $H\left(\varphi^{k}\right)$ converges to $\lim \inf H\left(\varphi^{k}\right)$ and that $\left(\varphi_{j}^{k}\right)$ converges to some $\varphi_{j}$ in $C^{0}(Z, \mathbb{R})$. Since the $\varphi_{j}$ 's sum to $\varphi$ and the $F_{j}$ 's are continuous, we get:

$$
H(\varphi) \leq \sum_{j=0}^{N} F_{j}\left(\varphi_{j}\right)=\lim \sum_{j=0}^{N} F_{j}\left(\varphi_{j}^{k}\right)=\liminf H\left(\varphi^{k}\right)
$$

\section{Proof of proposition 3}

Assertion 1. The fact that the infimum is attained in (18) follows at once from the weak-* compactness of $\Pi\left(\mu_{0}, \ldots, \mu_{N}\right)$ and the continuity of $\bar{c}$. Let $\nu \in \Delta(Z)$ and $\gamma_{j} \in \Pi\left(\mu_{j}, \nu\right)$ for every $j$. The disintegration theorem allows to write $\gamma_{j}=\gamma_{j}^{z} \otimes \nu$ (interpret the family $\gamma_{j}^{z}$ as conditional probabilities) for a measurable family of probabilities $\gamma_{j}^{z}$ on $X_{j}$. Now let $\eta \in \Delta(X \times Z)$ be defined for every $F \in C^{0}(X \times Z)$ by:

$$
\int_{X \times Z} F(x, z) d \eta(x, z):=\int F\left(x_{0}, \ldots ., x_{N}, z\right) d \gamma_{0}^{z}\left(x_{0}\right) \ldots . d \gamma_{N}^{z}\left(x_{N}\right) d \nu(z) .
$$

By construction, the marginal of $\eta$ on $X_{j} \times Z$ is $\gamma_{j}$ and defining $\gamma$ as the projection of $\eta$ on $X$ one has $\gamma \in \Pi\left(\mu_{0}, \ldots, \mu_{N}\right)$. Now we have:

$$
\begin{aligned}
\sum_{j=0}^{N} \int_{X_{j} \times Z} c_{j}\left(x_{j}, z\right) d \gamma_{j}\left(x_{j}, z\right) & =\int_{X \times Z} \sum_{j=0}^{N} c_{j}\left(x_{j}, z\right) d \eta(x, z) \\
& \geq \int_{X \times Z} \bar{c}(x) d \eta(x, z)=\int_{X} \bar{c}(x) d \gamma(x)
\end{aligned}
$$

Since $\nu$ and the $\gamma_{j}$ 's are arbitrary in the previous inequality and since $\gamma \in$ $\Pi\left(\mu_{0}, \ldots, \mu_{N}\right)$, we deduce that the value of $\left(\mathcal{P}^{*}\right)$ is greater than that of (18). 
Assertion 2. Let $\bar{\gamma}$ be a solution of (18) and define $\bar{\nu}:=\bar{z} \sharp \bar{\gamma}$ and $\gamma_{j}:=\left(\pi_{j}, \bar{z}\right) \sharp \bar{\gamma}\left(\right.$ where $\left.\pi_{j}(x):=x_{j}\right)$. Note that by construction $\gamma_{j} \in \Pi\left(\mu_{j}, \bar{\nu}\right)$. We then have:

$$
\begin{aligned}
\inf \left(\mathcal{P}^{*}\right) & \geq \int_{X} \bar{c}(x) d \bar{\gamma}(x)=\int_{X} \sum_{j=0}^{N} c_{j}\left(x_{j}, \bar{z}(x)\right) d \bar{\gamma}(x) \\
& =\sum_{j=0}^{N} \int_{X_{i} \times Z} c_{j}\left(x_{j}, z\right) d \gamma_{j}\left(x_{j}, z\right) \geq \sum_{j=0}^{N} W_{c_{j}}\left(\mu_{j}, \bar{\nu}\right) \geq \inf \left(\mathcal{P}^{*}\right) .
\end{aligned}
$$

This proves that the value of (18) is $\inf \left(\mathcal{P}^{*}\right)$ and that $\bar{\nu}=\bar{z} \sharp \bar{\gamma}$ solves $\left(\mathcal{P}^{*}\right)$.

Assertion 3. Finally assume that $\bar{\nu}$ solves $\left(\mathcal{P}^{*}\right)$, let $\bar{\gamma}_{j} \in \Pi\left(\mu_{j}, \bar{\nu}\right)$ be such that

$$
W_{c_{j}}\left(\mu_{j}, \bar{\nu}\right)=\int_{X_{j} \times Z} c_{j} d \bar{\gamma}_{j}
$$

Let us disintegrate each $\bar{\gamma}_{j}$ by writing $\bar{\gamma}_{j}:=\bar{\gamma}_{j}^{z} \otimes \bar{\nu}$ and define $\bar{\eta} \in \Delta(X \times Z)$ by:

$$
\int_{X \times Z} F(x, z) d \bar{\eta}(x, z):=\int F\left(x_{0}, \ldots, x_{N}, z\right) d \bar{\gamma}_{0}^{z}\left(x_{0}\right) \ldots d \bar{\gamma}_{N}^{z}\left(x_{N}\right) d \bar{\nu}(z)
$$

for all $F \in C^{0}(X \times Z)$. Finally denote by $\bar{\gamma}$ the projection of $\bar{\eta}$ on $X$. By construction, $\bar{\gamma} \in \Pi\left(\mu_{0}, \ldots, \mu_{N}\right)$ and thus the common value of $\left(\mathcal{P}^{*}\right)$ and (18) equals

$$
\begin{aligned}
\sum_{j=0}^{N} \int_{X_{j} \times Z} c_{j}\left(x_{j}, z\right) d \bar{\gamma}_{j}\left(x_{j}, z\right) & =\int_{X \times Z} \sum_{j=0}^{N} c_{j}\left(x_{j}, z\right) d \bar{\eta}(x, z) \\
& \geq \int_{X \times Z} \bar{c}(x) d \bar{\eta}(x, z)=\int_{X} \bar{c}(x) d \bar{\gamma}(x) .
\end{aligned}
$$

This proves on the one hand that $\bar{\gamma}$ solves (18) and on the other hand that for $\bar{\eta}$-a.e. $(x, z)$ one has

$$
\sum_{j=0}^{N} c_{j}\left(x_{j}, z\right)=\bar{c}(x)
$$

by continuity, this also implies that the support of $\bar{\eta}$ is included in the graph of $\bar{z}$. Hence for $\bar{\gamma}$-a.e. $x \in X$, the conditional probability $\bar{\gamma}^{x}$ is the Dirac mass at $\bar{z}(x)$, we thus have $\bar{\eta}=\left(\operatorname{id}_{X}, \bar{z}\right) \sharp \bar{\gamma}$ and, in particular $\bar{\nu}=\bar{z} \sharp \bar{\gamma}$. 


\section{References}

[1] G. Carlier, Duality and existence for a class of mass transportation problems and economic applications, Adv. in Math. Econ., vol. 5 (2003), pp. $1-21$.

[2] P.-A. Chiappori, R. J. McCann, L. Nesheim, Hedonic price equilibria, stable matching and optimal transport: equivalence, topology and uniqueness, preprint available at http://www.math.toronto.edu/ mccann/.

[3] I. Ekeland, An optimal matching problem, ESAIM Contrôle Optimal et Calcul des Variations, 11, 1, 57-71 (2005).

[4] I. Ekeland, R. Temam, Convex Analysis and Variational Problems, Classics in Mathematics, Society for Industrial and Applied Mathematics, Philadelphia, (1999).

[5] S.T. Rachev, L. Rüschendorf. Mass Transportation Problems. Vol. I: Theory; Vol. II : Applications, Springer-Verlag (1998).

[6] C. Villani, Topics in optimal transportation, Graduate Studies in Mathematics, 58, American Mathematical Society, Providence, RI, (2003). 